\title{
Degeneracy pressure of relic neutrinos and cosmic coincidence problem
}

\author{
Tae Hoon Lee \\ Department of Physics and Institute of Natural Sciences, \\ Soongsil University, Seoul 156-743 Korea \\ E-mail: thlee@ssu.ac.kr
}

\begin{abstract}
We consider the universe as a huge $\nu_{R}$-sphere formed with degenerate relic neutrinos and suggest that its constant energy density play a role of an effective cosmological constant. We construct the sphere as a bubble of true vacuum in a field theory model with a spontaneously broken $U(1)$ global symmetry, and we interpret the sphere-forming time as the transition time for recent acceleration of the universe. The coincidence problem may be regarded as naturally resolved in this model, because the relic neutrinos can make the $\nu_{R}$-sphere at the recent past time during the matterdominated era.
\end{abstract}

PACS numbers: 98.80.-k, 95.36.+x, 14.60.St 


\section{Introduction}

The inflationary big bang cosmology has been developed into a precision science by recent cosmological observations including supernova data [1] and measurements of cosmic microwave background radiation [2]. From those it is suggested that our universe is made up of about 74 percent dark energy as well as about 4 percent ordinary matter and about 22 percent dark matter. The observations triggered an explosion of recent interests in the origin of dark energy [3]. There are several approaches to understanding the dark energy such as quintessence [4] and phantom [5].

One of the simple ways to explain it is through an introduction of the cosmological constant $\Lambda$, which leads to an exponential expansion of the scale factor of the universe via $a(t) \sim e^{\sqrt{\Lambda / 3} t}$. Even though this simple form adequately describes current acceleration with a small value of $\Lambda$, it cannot say anything about important issues including the coincidence problem [6]. Why in the recent past the expansion of the universe started to accelerate? In this article, we suggest relic neutrinos, which are the lightest among all known fermionic particles and thus have become non-relativistic in the last cosmic time, might be related with this issue. We study a possible role of degeneracy pressure of the neutrinos in the evolution of the universe after neutrino decoupling $t_{d}$.

When we consider a sphere of radius $R(t)$ containing all relic neutrinos (of total number $\left.N(t)=M(t) / m_{\nu}\right)$ at a cosmic time $t\left(\ll t_{d}\right)$, the balance between degeneracy energy of the non-relativistic neutrinos, $\hbar^{2} M^{5 / 3} /\left(m_{\nu}^{8 / 3} R^{2}\right)$, and their gravitational energy, $G M^{2} / R$, gives us such relation of the radius as $R(t)=\hbar^{2} /\left(G m_{\nu}^{8 / 3} M^{1 / 3}(t)\right)$. On the other hand, the balance between degeneracy energy of relativistic neutrinos, $\hbar c M^{4 / 3} /\left(m_{\nu}^{4 / 3} R\right)$, and the gravitational energy yields the maximum total mass [7] $M_{c}=\left(\frac{\hbar c}{G}\right)^{\frac{3}{2}} m_{\nu}^{-2}$. From these the radius of the sphere is bounded below,

$$
R>R_{c}\left(\simeq \frac{\hbar^{2}}{c^{2} m_{\nu}^{2} l_{p}}\right) \sim 10^{23} \times\left(\frac{1 e V}{m_{\nu} c^{2}}\right)^{2} \mathrm{~cm}
$$

with Planck length $l_{p}$.

The matter-dominated era of our universe began at $t_{m}=70 \mathrm{Kyr}$ and ended at the transition time $t_{t}=5 \mathrm{Gyr}$ [8], at which the universe started to accelerate. In the era $t_{m}<t<t_{t}$, we can neglect the pressure of the other matter than the degeneracy pressure of relic neutrinos caused by the Heisenberg uncertainty principle. If we assume that at a cosmic time $t=t_{\chi}$ a huge bubble of relic neutrinos (which we call a $\nu_{R}$-sphere) is formed with a radius $R_{\chi}$ determined from the condition $R_{\chi}\left(t_{\chi}\right)=R_{c}$ as a gigantic Fermion star [9], then the universe's main-frame in the time can be imagined as made up of these relic neutrinos filled up to the Fermi energy. Comparing the horizon radius of the present universe $R_{\text {now }} \sim 10^{29} \mathrm{~cm}$ (or $R_{m} \sim 10^{27} \mathrm{~cm}$ at $t=t_{m}$ ) with the critical value $R_{c}\left(\simeq R_{\chi}\right)$ in equation (1), we see that these values are not different from each other if we take $m_{\nu} \sim 1-10^{-3} \mathrm{eV}$.

From the time $t \simeq t_{\chi}$, the universe has a total energy density contributed mainly from the $\nu_{R^{-}}$sphere with a (effective) cosmological constant $\rho_{\nu}\left(\simeq M_{c} / R_{c}^{3}\right)$ in addition to cold dark matter $\rho_{C D M}\left(\propto R^{-3}\right)$, and thus the time $t_{\chi}$ can be interpreted as the 
transition time $t_{t}$. We think that the coincidence problem might be naturally resolved because neutrinos become relic in this matter-dominated era and can make the $\nu_{R}$-sphere at the recent past time $t_{\chi}\left(<t_{\text {now }}\right)$. In Section 2 we demonstrate a realization of this idea in a global $U(1)$ symmetric field theory model with a complex scalar field and relic neutrinos in curved spacetime. Section 3 contains summary and discussions.

\section{A Global U(1) Model}

\subsection{General Relativistic Formulation}

The action of a global $U(1)$ symmetric model of complex scalar $\Phi$ and neutrino fields $\Psi$ minimally coupled to gravity is given by $S_{m}=\int d^{4} x \sqrt{-g} \mathcal{L}_{\text {matter }}$, where

$$
\begin{aligned}
\mathcal{L}_{\text {matter }} & =-g^{\mu \nu} \partial_{\mu} \Phi^{\dagger} \partial_{\nu} \Phi-V\left(\Phi^{\dagger} \Phi\right) \\
& +\frac{i}{2}\left(\bar{\Psi} \gamma^{a} \nabla_{a} \Psi-\nabla_{a} \bar{\Psi} \gamma^{a} \Psi\right)-m_{0} \bar{\Psi} \Psi-q \Phi^{\dagger} \Phi \bar{\Psi} \Psi
\end{aligned}
$$

with a bare mass of neutrino $m_{0}$. The last term describes the scalar-neutrino interaction preserving the $U(1)$ symmetry with a parameter $q$ of dimension, for example, $1 / M_{p}$ (Planck mass $M_{p}$ ) in gravity-induced $U(1)$ breaking models [10, 11].

From the above action, we obtain following equations for scalar field and neutrino.

$$
\begin{aligned}
& \frac{1}{\sqrt{-g}} \partial_{\mu}\left(\sqrt{-g} g^{\mu \nu} \partial_{\nu} \Phi\right)-\frac{\partial V}{\partial \Phi^{\dagger}}-q \Phi \bar{\Psi} \Psi=0, \\
& i \gamma^{a} \nabla_{a} \Psi-m_{0} \Psi-q \Phi^{\dagger} \Phi \Psi=0,
\end{aligned}
$$

where the $\gamma^{a}$-matrices satisfy the Clifford algebra in a locally flat inertial coordinate, $\left\{\gamma^{a}, \gamma^{b}\right\}=-2 \eta^{a b}$ with $\eta^{a b}=\operatorname{Diag}(-1,1,1,1)$, and the covariant derivative

$$
\nabla_{a}=e^{\mu}{ }_{a}\left(\partial_{\mu}+\Gamma_{\mu}\right)
$$

is constructed from the vierbein $e^{\mu}{ }_{a}$ and spin connection $\Gamma_{\mu}[12$. The energy-momentum tensor in this model

$$
\begin{array}{r}
T_{\mu \nu}=2 \partial_{\mu} \Phi^{\dagger} \partial_{\nu} \Phi-g_{\mu \nu}\left[\partial^{\beta} \Phi^{\dagger} \partial_{\beta} \Phi+V\left(|\Phi|^{2}\right)\right] \\
-\frac{i}{4}\left[\left(\bar{\Psi} \gamma_{\mu} \nabla_{\nu} \psi-\nabla_{\nu} \bar{\Psi} \gamma_{\mu} \Psi\right)+(\mu \leftrightarrow \nu)\right]
\end{array}
$$

is obtained by using the standard definition $T_{\mu \nu} \equiv-\frac{2}{\sqrt{-g}} \frac{\delta S_{m}}{\delta g^{\mu \nu}}=-\frac{e_{a \mu}}{\operatorname{det}\{e\}} \frac{\delta S_{m}}{\delta e_{a}^{\nu}}$. In next subsection we construct a concrete model with a specific potential of the scalar field.

\subsection{A Bubble Universe with U(1) Symmetry Spontaneously Broken}

Assuming a potential of the scalar field

$$
V\left(|\Phi|^{2}\right)=\lambda|\Phi|^{2}\left(|\Phi|^{2}-v^{2}\right)^{2}
$$

with dimensionful constants $\lambda$ and $v$, we consider a spherical bubble of true vacuum $\langle\Phi\rangle=v$ with a spontaneously broken $U(1)$ global symmetry, immersed in the different (false) vacuum state $\langle\Phi\rangle=0$ having the $U(1)$ symmetry. To construct a field theoretical 
model in which the ideas discussed in the (previous) Section 1 can be simply realized, we adopt the Thomas-Fermi(TF) approximation [9, 13, 14] inside the bubble including all relic neutrinos of the total number $N=\int d^{3} x \sqrt{-g} \Psi^{\dagger} \Psi e^{t}{ }_{0}$ with $e_{0}^{t}=\frac{1}{\delta \alpha}$. The neutrinos have the effective mass, $m_{e f f}=q v^{2}$ with $\langle\Phi\rangle=v$, and we put $m_{0}=0$ from now on for the simplicity. In the TF approximation there is at each point in space a Fermi sea of massive neutrinos with the local Fermi momentum $q_{F}(r)$, and the number density of neutrinos is given by

$$
\left\langle\Psi^{\dagger} \Psi\right\rangle_{T F}=\frac{2}{(2 \pi)^{3}} \int d^{3} q n_{q}=\frac{q_{F}{ }^{3}(r)}{3 \pi^{2}}
$$

with the Fermi distribution $n_{q}=\theta\left(q_{F}-q\right)$. From Eq. (8) and Dirac's equation, $i \partial_{t} \Psi=E \Psi$, we have the energy density of neutrinos

$$
\left\langle\frac{i}{2 \delta \alpha}\left(\bar{\Psi} \gamma^{0} \partial_{t} \Psi-\partial_{t} \bar{\Psi} \gamma^{0} \Psi\right)\right\rangle_{T F}=\frac{2}{(2 \pi)^{3}} \int d^{3} q n_{q} E(q) e_{0}^{t} \equiv \rho(r) .
$$

The pressure $p(r)$ of neutrinos is given in equation (13) below.

When we express the spherically symmetric (static) spacetime as

$$
d s^{2}=-\delta^{2}(r) \alpha^{2}(r) d t^{2}+\frac{d r^{2}}{\alpha^{2}(r)}+r^{2} d \theta^{2}+r^{2} \sin ^{2} \theta d \phi^{2},
$$

the (background) scalar field equation (3) reads

$$
\frac{1}{r^{2} \delta}\left(r^{2} \delta \alpha^{2} \Phi^{\prime}\right)^{\prime}-\lambda \Phi\left(|\Phi|^{2}-v^{2}\right)\left(3|\Phi|^{2}-v^{2}\right)-q \Phi\langle\bar{\Psi} \Psi\rangle_{T F}=0
$$

and the Einstein equation, $G_{\mu \nu}=\kappa T_{\mu \nu}$ with $\kappa=8 \pi G$, in this TF approximation, yields

$$
\begin{aligned}
& \frac{\left(1-\alpha^{2}\right)}{r^{2}}-\frac{1}{r}\left(\alpha^{2}\right)^{\prime}=\kappa\left[\alpha^{2}\left|\Phi^{\prime}\right|^{2}+V+\frac{i}{2 \delta \alpha}\left\langle\bar{\Psi} \gamma^{0} \partial_{t} \Psi-\partial_{t} \bar{\Psi} \gamma^{0} \Psi\right\rangle_{T F}\right], \\
- & \frac{\left(1-\alpha^{2}\right)}{r^{2}}+\frac{\left(\delta^{2} \alpha^{2}\right)^{\prime}}{r \delta^{2}}=\kappa\left[\alpha^{2}\left|\Phi^{\prime}\right|^{2}-V-\frac{i \alpha}{2}\left\langle\bar{\Psi} \gamma^{1} \partial_{r} \Psi-\partial_{r} \bar{\Psi} \gamma^{1} \Psi\right\rangle_{T F}\right], \\
& \frac{1}{2 \delta^{2}}\left(\delta^{2} \alpha^{2}\right)^{\prime \prime}-\frac{\left(\delta^{2} \alpha^{2}\right)^{\prime}}{4 \delta^{2}} \frac{\left(\delta^{2}\right)^{\prime}}{\delta^{2}}+\frac{\left(\delta^{2} \alpha^{2}\right)^{\prime}}{r \delta^{2}}-\frac{\alpha^{2}}{2 r} \frac{\left(\delta^{2}\right)^{\prime}}{\delta^{2}} \\
= & \kappa\left[-\alpha^{2}\left|\Phi^{\prime}\right|^{2}-V-\frac{i}{2 r}\left\langle\bar{\Psi} \gamma^{2} \partial_{\theta} \Psi-\partial_{\theta} \bar{\Psi} \gamma^{2} \Psi\right\rangle_{T F}\right] \\
& \frac{1}{2 \delta^{2}}\left(\delta^{2} \alpha^{2}\right)^{\prime \prime}-\frac{\left(\delta^{2} \alpha^{2}\right)^{\prime}}{4 \delta^{2}} \frac{\left(\delta^{2}\right)^{\prime}}{\delta^{2}}+\frac{\left(\delta^{2} \alpha^{2}\right)^{\prime}}{r \delta^{2}}-\frac{\alpha^{2}}{2 r} \frac{\left(\delta^{2}\right)^{\prime}}{\delta^{2}} \\
= & \kappa\left[-\alpha^{2}\left|\Phi^{\prime}\right|^{2}-V-\frac{i}{2 r}\left\langle\frac{1}{\sin \theta}\left(\bar{\Psi} \gamma^{3} \partial_{\phi} \Psi-\partial_{\phi} \bar{\Psi} \gamma^{3} \Psi\right)\right\rangle_{T F}\right],
\end{aligned}
$$

where $\Phi^{\prime} \equiv \frac{\partial \Phi}{\partial r}, \ldots$, have been used for the simplicity. The results in above equation (12) are consistent with those in the case of fermion stars [9], when

$$
\begin{aligned}
& \left\langle\frac{\alpha}{2 i}\left(\bar{\Psi} \gamma^{1} \partial_{r} \Psi-\partial_{r} \bar{\Psi} \gamma^{1} \Psi\right)\right\rangle_{T F}=\left\langle\frac{1}{2 i r}\left(\bar{\Psi} \gamma^{2} \partial_{\theta} \Psi-\partial_{\theta} \bar{\Psi} \gamma^{2} \Psi\right)\right\rangle_{T F} \\
& =\left\langle\frac{1}{2 i r \sin \theta}\left(\bar{\Psi} \gamma^{3} \partial_{\phi} \Psi-\partial_{\phi} \bar{\Psi} \gamma^{3} \Psi\right)\right\rangle_{T F}=p(r),
\end{aligned}
$$


are assumed in the spherically symmetric spacetime, and from Dirac's equation and its Hermitian conjugate we have the relation $\rho(r)-3 p(r)=q v^{2}\langle\bar{\Psi} \Psi\rangle_{T F}$.

Now assume that there exist regular solutions of the metric components, scalar field, and the energy density and pressure of neutrinos, and we get approximate solutions to above equations (11)-(12) as

$$
\begin{aligned}
\Phi(r) & =v+\frac{\rho_{0}-3 p_{0}}{6} r^{2}+\mathcal{O}\left(r^{3}\right), \\
\alpha^{2} & =1-\frac{\kappa}{3} \rho_{0} r^{2}+\mathcal{O}\left(r^{3}\right), \\
\delta^{2} \alpha^{2} & =\left\{1+\kappa\left(\frac{1}{6} \rho_{0}+\frac{1}{2} p_{0}\right) r^{2}\right\}+\mathcal{O}\left(r^{3}\right),
\end{aligned}
$$

with $\rho_{0} \equiv \rho(0)$ and $p_{0} \equiv p(0)$. These solutions are consistent with the results in fermionic stars with a global monopole [14]. On the other hand, to equation (12) with $V \simeq 0, \rho \simeq 0$ and $p \simeq 0$ far away from the horizon of the true vacuum bubble $r_{h} \equiv \sqrt{1 / \kappa \rho_{0}} \ll r$, solutions of the metric components in equation (10) can be taken asymptotically as $\alpha^{2} \simeq 1-\frac{2 \kappa M}{r}$ and $\delta^{2} \simeq 1$ with the total mass of all particles inside the bubble universe $M$. With the metric components we have the asymptotic solution $\Phi \simeq r^{-2} e^{-\sqrt{\lambda} v^{2} r}$. Under the condition

$$
\sqrt{\lambda} v^{2} \kappa M=1
$$

the solution for $\Phi$ satisfies all the relevant equations even upto next leading order.

From the effective neutrino mass $m_{e f f}=q v^{2}$ inside the bubble, we can estimate the true vacuum expectation value as $v^{2} \sim m_{\text {eff }} M_{p}$, which gives us $\lambda \simeq M_{p}^{4} /\left(v^{4} M^{2}\right) \sim$ $10^{-120} / m_{\text {eff }}^{2}$ when equation (15) is used. On the other hand, if the potential $V\left(|\Phi|^{2}\right)$ of the scalar field is induced by gravity [10, 11] with the dimensionful constant $\lambda \simeq v^{n-2} / M_{p}^{n}$ for $0 \leq n$, then its magnitude comparable with the above is given as $\lambda \sim 10^{-112} \times m_{\text {eff }}^{2} /(1 \mathrm{eV})^{4}$ for $n=6$, which is the same as $10^{-120} / m_{\text {eff }}^{2}$ if $m_{\text {eff }} \sim 10^{-2} \mathrm{eV}$.

\section{Summary and Discussions}

Considering a huge $\nu_{R}$-sphere to be possibly formed at $t=t_{\chi}$, with degenerate relic neutrinos filled up to the Fermi energy, we suggest that its constant energy density $\rho_{\nu}$ play a role of an effective cosmological constant. The time $t_{\chi}$ can be interpreted as the transition time $t_{t}$, and these lead to the interesting possibility that our model gives one way of understanding the coincidence problem [6], because the relic neutrinos can make the $\nu_{R}$-sphere at the recent past time $t_{\chi}\left(<t_{\text {now }}\right)$ during the matter-dominated era. In the Section 2 we have constructed a spontaneously broken $U(1)$ global symmetric model, in which the energy density $T_{t t} \equiv \rho\left(\sim \rho_{0}\right)$ in the right hand side of the first equation of (12) can be put as $\rho_{\nu} \sim M_{c} / R_{c}^{3}$ of the $\nu_{R}$-sphere considered from Section 1 . However the formulation was constrained in a static case and further study is necessary on the cosmic time evolution of the scale factor $R(t)$. The predicted values of the relic 
neutrino mass, in the range $m_{\nu} \sim 1-10^{-3} \mathrm{eV}$ given both in Section 1 and the last line of Section 2, are anticipated to be tested by future observations.

We would like to make a few comments. In future works, we need a fully relativistic treatment (and possible numerical methods) in calculation of the more rigorous value of the critical radius of the $\nu_{R}$-sphere in equation (11). For our $U(1)$ model to work with the appropriate (relic) neutrino mass, the parameter $\lambda$ in the potential of the scalar field in equation (17) should be very small, which might be realized in some models using an extremely light scalar field [15] or in gravity-induced models [10, 11]. We are seeking to find others models related with ours, in which the global $U(1)$ symmetry is

spontaneously broken with $\langle\Phi\rangle \equiv v=\sqrt{m_{\text {eff }} M_{p}} \sim 100 \mathrm{TeV}$. During a very slow phase transition $(\lambda \ll 1)$ from the false vacuum with $\langle\Phi\rangle=0$ to the true vacuum with $\langle\Phi\rangle=v$, masses of neutrinos look changing from from 0 to $m_{\text {eff }}=q v^{2}$, and the $\nu_{R^{-}}$sphere made of the massive neutrinos could play a similar role to a effective cosmological constant in our model, while a growing matter component (neutrinos) in Ref. [16], instead, helps such a field as the cosmon [16] responsible for the dark energy.

\section{Acknowledgements}

This research was supported by the Basic Science Research Program through the National Research Foundation of Korea(NRF) funded by Ministry of Education, Science and Technology(2010-0012692).

\section{References}

[1] Perlmutter S J et al. 1999 Astrophys. J. 517 565; Riess A G et al. 2007 Astrophys. J. 659 98, astro-ph/0611572

[2] Spergel D N et al. (WMAP Collaboration) 2007 Astrophys. J. Suppl. 170 377, astro-ph/0603449

[3] Perlmutter S J, Turner M S and White M J 1999 Phys. Rev. Lett. 83 670, astro-ph/9901052

[4] Caldwell R R, Dave R and Steinhardt P J 1998 Phys. Rev. Lett. 80 1582, astro-ph/9708069, and references therein

[5] Caldwell R R 2002 Phys. Lett. B 545 23, astro-ph/9908168, Hong S T, Lee J, Lee T H and Oh P 2008 Phys. Rev. D 78 047503, arXiv:0801.3781 [gr-qc]

[6] Chimento L P, Jakubi A S, Pavon D and Zimdahl W 2003 Phys. Rev. D 67 083513, astro-ph/0303145

[7] Chandrasekhar S 1931 Astrophys. J. 7481

[8] Hartnett J G and Oliveira F J 2006 Found. Phys. Lett. 19 519, astro-ph/0603500

[9] Lee T D and Pang Y 1987 Phys. Rev. D 353678

[10] Barbieri R, Ellis J R, and Gaillard M K 1980 Phys. Lett. B 90249

[11] Akhmedov E, Berezhiani Z, and Senjanovic G 1992 Phys. Rev. Lett. 693013

[12] Lee T H and McKellar B H J 2003 Phys. Rev. D 67103007

[13] Bachall S, Lynn B W and Selipsky S B 1989 Nucl. Phys. B 325606

[14] Li X Z and X. H. Zhai X H 1995 Phys. Lett. B 364212

[15] He X G, Mckellar B H J and Stephenson Jr. G J 2004 Phys. Lett. B 444 75, Erratum-ibid. B 581 270 (2004), hep-ph/9807338

[16] Amendola L, Baldi M, and Wetterich C 2008 Phys. Rev. D 78 023015, arXiv:0706.3064 [astro-ph] 